\title{
Algebraic Current-voltage and Voltage Dependent Resistance Expressions for Ballistic Nano Con- ductors and Their Low Voltage Nonlinearity
}

\author{
Serhan YAMACLI ${ }^{1,2, *}$
}

(Received 7 June 2013; accepted 1 July 2013; published online 25 July 2013)

\begin{abstract}
In this study, an algebraic current-voltage $(I-V)$ equation suitable for the hand-calculation of ballistic nano conductors is derived from Landauer's formulation. A voltage and temperature dependent resistance expression is also obtained. It is shown that the presented algebraic $I-V$ expression and the original Landauer's formula give the same characteristics as expected. Moreover, the $I$ - $V$ characteristics of ballistic nano conductors are investigated and it is concluded that there is an inescapable nonlinearity originating from the curvature of Fermi-Dirac distribution function in low voltage range. Finally, the total harmonic distortion (THD) of a sample ballistic nano conductor caused from its low voltage nonlinearity is computed via HSPICE simulations.
\end{abstract}

Keywords: Landauer's formula; Ballistic nano conductors; Nonlinearity

Citation: Serhan YAMACLI, "Algebraic Current-voltage and Voltage Dependent Resistance Expressions for Ballistic Nano Conductors and Their Low Voltage Nonlinearity", Nano-Micro Lett. 5(3), 169-173 (2013). http://dx.doi.org/10.5101/nml.v5i3.p169-173

\section{Introduction}

Nano conductors such as metallic carbon nanotubes (CNT) and graphene nano ribbon (GNR) transmission lines are taking great attention due to the limitations of the conventional copper and aluminium interconnect technology $[1,2]$. Several models for new generation nano conductors exist in the literature $[3,4,5]$ since these models are obviously needed for their design and analysis. In all of these models, current-voltage $(I-V)$ variations of nano conductors are clearly the main characteristics that have to be modelled accurately $[6,7]$.

$I-V$ characteristics of nano conductors are obtained using Landauer's formulation $[6,8,9]$ which is proven to be accurate in the literature since it is compatible with the experimental data for nano conductors such as metallic CNTs [10], graphene sheets [11] and gold nanowires [12]. Although Landauer's formula accurately describes the $I-V$ characteristics of nano conductors, it is an equation containing integral calculations. An algebraic expression that does not employ any integration suitable for faster hand-calculation of the $I-V$ characteristics does not exist in the literature.

In this study, an algebraic equation without integration is derived for ballistic nano conductors to calculate their $I-V$ characteristics. The $I-V$ characteristics obtained using the proposed algebraic $I-V$ expression is compared to the results of the original Landauer's formula to verify its accuracy. A voltage and temperature dependent resistance expression is also derived. Then, $I-V$ characteristics of nano conductors at low voltages are discussed. It is concluded that ballistic nano conductors show nonlinearity at low voltages and this nonlinearity is caused from the curvature of the FermiDirac distribution function hence it is inescapable. Fi-

\footnotetext{
${ }^{1}$ Creighton Technologies Ltd., 145-157 St. John Street, EC1V 4PW, London, UK

${ }^{2}$ Nuh Naci Yazgan University, Faculty of Engineering, Department of Electrical-Electronics Engineering, 38400, Kocasinan, Kayseri, Turkey

*Corresponding author. E-mail: syamacli@nny.edu.tr
} 
nally, the total harmonic distortion (THD) introduced by a sample ballistic nano conductor originating from its low voltage nonlinearity is obtained by HSPICE simulations.

\section{Presented $I-V$ expression for ballistic nano conductors}

The current passing through the nano conductor as shown in Fig. 1 is calculated by Landauer's formula shown in Eq. (1) $[6,8,9]$.

$$
I=\frac{2 q}{h} \int_{\mu_{1}}^{\mu_{2}} \underbrace{\frac{2 \pi \gamma_{1} \gamma_{2}}{\gamma_{1}+\gamma_{2}} D O S(E)}_{T(E)}\left[f_{1}(E)-f_{2}(E)\right] d E
$$

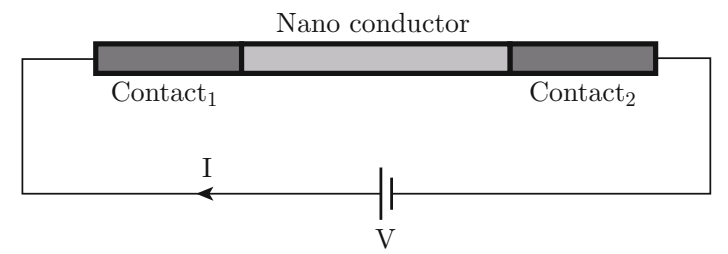

Fig. 1 A nano conductor with contacts and the scattering region.

In Eq. (1), $\mu_{1}$ and $\mu_{2}$ are the electrochemical potentials with $\mu_{2}=\mu_{1}-q V ; \gamma_{1}$ and $\gamma_{2}$ are the broadenings of contact $_{1}$ and contact $_{2}$, respectively; $q$ is the elementary charge; $h$ is Planck's constant; $D O S(E)$ is the density of states (DOS) function of the nano conductor; $\operatorname{Tr}(E)$ is the energy dependent transmission of electrons $[8,9]$; $f_{1}(E)$ and $f_{2}(E)$ are Fermi-Dirac electron distribution functions of contact $_{1}$ and contact $_{2}$ which can be given as in Eq. (2) and Eq. (3), respectively [9].

$$
\begin{gathered}
f_{1}(E)=\frac{1}{1+e^{\frac{E-\mu}{k_{B} T}}} \\
f_{2}(E)=\frac{1}{1+e^{\frac{E-\mu+q V}{k_{B} T}}}
\end{gathered}
$$

where $\mu$ is the equilibrium electrochemical potential of the nano conductor; $k_{B}$ is Boltzmann's constant and $T$ is the absolute temperature. Various nano conductors have constant valued $D O S$ functions in the energy range of interest $[7,13,14,15]$ hence by taking the DOS function of Eq. (1) as constant and evaluating the integral, an $I-V$ expression without integral calculation for nano conductors is derived as shown in Eq. (4). In this equation, DOS represents the density of states value and the term shown as $T_{r}$ is the transmission of electrons. Note that $T_{r}=1$ for ballistic nano conductors.

$$
I=\frac{2 q k_{B} T}{h} \underbrace{\frac{2 \pi D O S \gamma_{1} \gamma_{2}}{\gamma_{1}+\gamma_{2}}}_{T r} \times
$$

$$
\left[\ln \left(1+\exp \left(\frac{-q V}{k_{B} T}\right)\right)-\ln \left(\frac{4}{1+\exp \left(\frac{q V}{k_{B} T}\right)}\right)\right]
$$

The importance of the presented $I$ - $V$ expression shown in Eq. (4) is that it is an algebraic equation without any integration which enables easy calculation of $I-V$ characteristics of ballistic nano conductors. The derived formula is especially useful for fast hand-calculation.

In addition, $I-V$ characteristics of a ballistic nano conductor $(T r=1)$ is obtained using both Landauer's formula and the presented simplified expression to verify its accuracy. The calculations are performed for three different temperatures: $200 \mathrm{~K}, 300 \mathrm{~K}$ and $400 \mathrm{~K}$. Obtained $I-V$ characteristics are shown in Fig. 2. As it can be seen from Fig. 2, the original Landauer's formula and the presented $I-V$ expression without integration give the same characteristics validating the presented $I$ - $V$ formula. Note that the accuracy of the $I-V$ characteristics obtained using Landauer's formula is experimentally verified for numerous nano conductors in the literature $[10,11,12]$ which implies that the results of the presented formula are also compatible with experimental measurements.

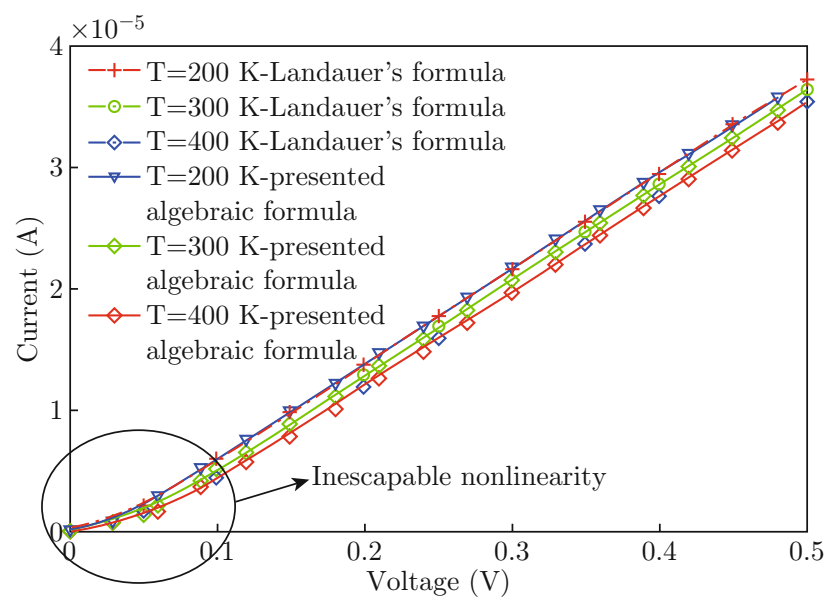

Fig. $2 \quad I-V$ characterisation of ballistic nano conductors obtained using Landauer's formula and the proposed algebraic $I-V$ expression for $\mathrm{T}=200 \mathrm{~K}, \mathrm{~T}=300 \mathrm{~K}$ and $\mathrm{T}=400 \mathrm{~K}$.

There is an important fact observed from the $I-V$ characteristics of ballistic nano conductors. As it can be seen from Fig. 2, nano conductors having constant DOS in the energy range of interest show nonlinear $I$ - $V$ characteristics at lower voltages even in the ballistic transport regime. This nonlinearity in the $I-V$ characteristics is caused from the shape of Fermi-Dirac distribution functions. At nonzero temperatures, the edges of Fermi-Dirac distribution functions are not abrupt but curved and this curvature causes the nonlinearity in the $I-V$ characteristics. The edges of the Fermi-Dirac distribution functions of all nano conductor materials are 
curved at nonzero temperatures; hence the nonlinearity caused from this fact is an inescapable nonlinearity.

This result is interesting and useful since ballistic nano conductors such as metallic nanowires and carbon nanotubes are promising candidates to be used as interconnects, where the design engineer needs to have accurate descriptions of their $I-V$ characteristics. It is worth noting that the mean free path in metallic carbon nanotubes are measured as $1.5 \mu \mathrm{m}$ [16] while the mean free path of electrons in GNRs and gold nanowires are given as $100 \mathrm{~nm}$ and $50 \mathrm{~nm}$, respectively [17,18]. Considering the expected interconnect lengths in the ITRS roadmap [19], nano interconnects can be used in the ballistic regime.

In the literature, ballistic nano conductors are considered to have a constant resistance value in the ballistic transport regime and consequently analysed with linear $I-V$ characteristics $[6,8,10]$. This approach is valid for low absolute temperature values where the Fermi-Dirac electron distribution functions of the contacts given in Eq. (2) and Eq. (3) are abrupt enough to be taken as step functions. However, for higher temperatures such as room temperature, the edges of the Fermi-Dirac functions of contacts become curved and this leads to the inescapable nonlinear $I$ - $V$ characteristics which is modelled in Eq. (4) and shown in Fig. 2. This is another result of this study.

\section{Voltage dependent resistance of ballistic nano conductors}

A voltage and temperature dependent resistance expression for ballistic nano conductors does not exist in the literature and it is also obtained in this study. The resistance formula is derived using the straightforward derivation procedure from Eq. (4) and given in Eq. (5).

$$
R(V, T)=\frac{\partial V}{\partial I}=\underbrace{\frac{h}{2 q^{2}} \underbrace{\frac{\left(\gamma_{1}+\gamma_{2}\right)}{2 \pi D O S \gamma_{1} \gamma_{2}}}_{1 / T r}}_{R_{n}} \underbrace{\left[\frac{1+\exp \left(\frac{q V}{k_{B} T}\right)}{-1+\exp \left(\frac{q V}{k_{B} T}\right)}\right]}_{f(V, T)}
$$

The resistance expression shown in Eq. (5) is composed of a nominal resistance value, $R_{n}$, multiplied by a voltage and temperature dependent term, $f(V, T)$. As the applied voltage $V$ increases, $f(V, T)$ approaches unity and the nanodevice resistance takes its nominal value $R_{n} . R_{n}$ also consists of the multiplication of two terms: the first term is the universal resistance quantum value, $R_{Q}$, having value of $12.9 \mathrm{k} \Omega$ and the second term $1 / T_{r}$, which is the inverse of the transmission coefficient of the electrons. For ballistic nano conductors $T_{r}=1$ and in this case when $f(V, T)$ also approaches unity, nanodevice resistance $R(V, T)$ approaches $R_{Q}$, which is its minimum value as verified by various experiments $[20,21,22]$. Although constant resistance values of ballistic nano conductors are extensively studied in the literature [23,24], a resistance expression dependent on both the applied voltage and the temperature is given for the first time in this paper as in Eq. (5) to the knowledge of the authors.

The dependency of the resistance on the applied voltage is plotted in Fig. 3 at different temperatures for a sample ballistic nano conductor using the proposed resistance equation. Note that $R_{n}=R_{Q}$ for this example since $T_{r}$ is unity for ballistic nano conductors. As it can be seen from Fig. 3, at low temperatures the resistance of the nano conductor takes its nominal value for even low voltages. It is because the Fermi-Dirac electron distribution functions of the contacts have sharp edges at low temperatures. However, as the temperature increases, since the edges of the Fermi-Dirac functions of the contacts become curved, the resistance value becomes highly dependent on the applied voltage for low voltages. Note that the nonlinearity of nano conductors at low voltages is caused from the $f(V, T)$ term in Eq. (5) hence the low voltage nonlinearity always exists independent of nano conductor type, geometry and ballistic or non-ballistic transport regimes. In other words, low voltage nonlinearity of nano conductors applies to all nano conductor types since the electron transport of all these devices are modelled with Fermi-Dirac distribution functions that produce the voltage and temperature dependent term $f(V, T)$.

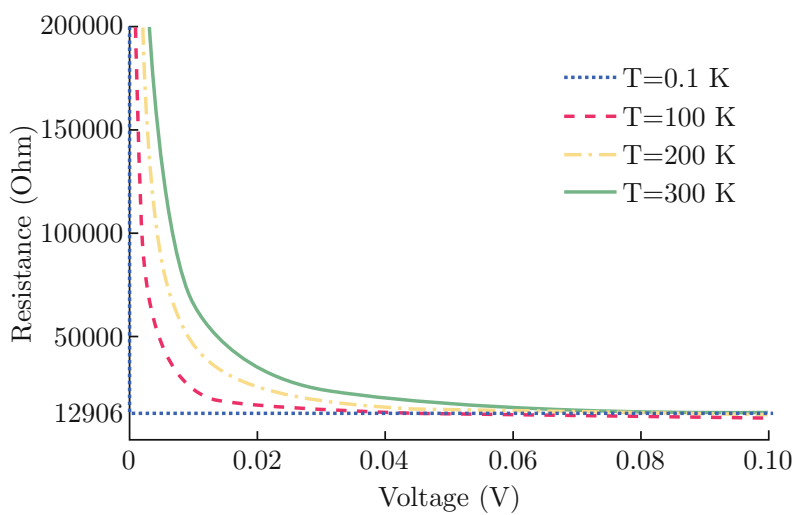

Fig. 3 Variation of the resistance of a ballistic nano conductor with the applied voltage for various temperatures.

\section{Effects of low voltage nonlinearity of ballistic nano conductors on the signal trasport}

Considering that the threshold voltage of integrated circuit (IC) technology is expected to be as low as 0.08 $\mathrm{V}$ in a few years according to the ITRS roadmap [19], the voltage dependency of the resistance shown in Fig. 3 is important since a considerable amount of nonlinear- 
ity is added at low voltage range. In order to visualize the effects of nonlinear resistance, the signal transport of a ballistic nano conductor shown in the inset of Fig. 4 is simulated in HSPICE using the $R(V, T)$ expression of Eq. (5). $R(V, T)$ expression is imported as a Verilog-A model into HSPICE with $T_{r}=1$. The input and output voltage signals are plotted in Fig. 4. As it can be seen from Fig. 4, ballistic nano conductor adds considerable distortion to the transported signals at low voltages.

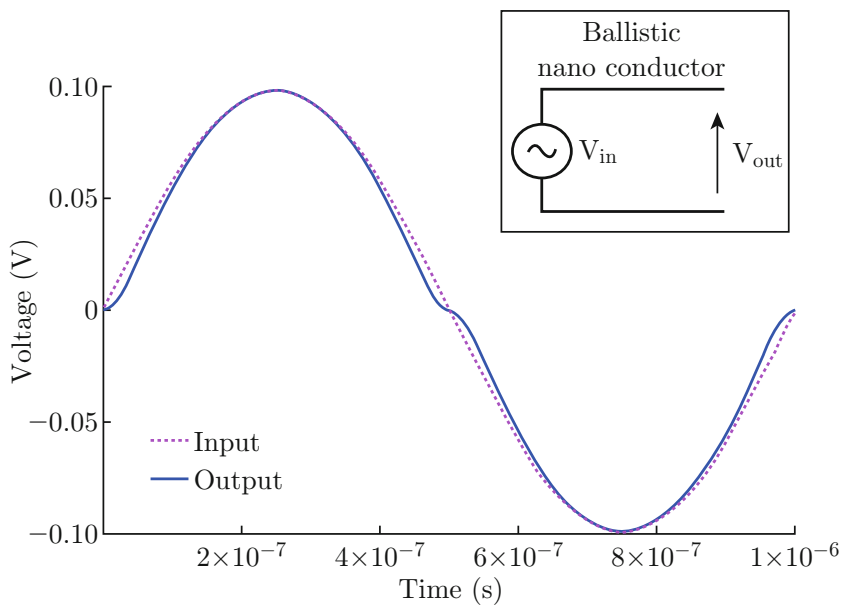

Fig. 4 A ballistic nano conductor used for signal transport (inset) and its input and output voltage waveforms.

Table 1 THD introduced by the ballistic nano conductor $\left(T_{r}=1\right)$ for various signal amplitudes at $1 \mathrm{MHz}$.

\begin{tabular}{ccccc}
\hline Signal amplitude (V) & 0.03 & 0.05 & 0.08 & 0.1 \\
\hline THD (\%) & 3.36 & 2.51 & 1.44 & 0.8 \\
\hline
\end{tabular}

The total harmonic distortion (THD) introduced by the nonlinearity of a sample ballistic nano conductor $\left(T_{r}=1\right)$ is also obtained by HSPICE and given in Table 1 for various signal amplitudes. The nonlinearity and the distortion caused from the curvature of the Fermi-Dirac distribution functions for ballistic nano conductors decrease when the voltage amplitude increases as it should be according to Eq. (5) and Fig. 3. Note that this behaviour is the opposite of the nonlinear behaviour caused from electron-phonon scatterings of non-ballistic nano conductors. In non-ballistic nano conductors, as the voltage increases, the electron-phonon scatterings increase causing an increment in the nonlinearity and distortion. However for ballistic conductors, the nonlinearity is not caused from electron-phonon scatterings but from the curvature of the Fermi-Dirac distribution function which is more effective at lower voltages. In other words, this type of nonlinearity of ballistic nano conductors is higher at lower voltages which are also verified by the values shown in Table 1 .

\section{Conclusions}

The results of this study are: i) an algebraic $I-V$ expression without integration for nano conductors is proposed which is suitable for fast computer and hand calculation. The accuracy of the presented equation is verified by comparison to the original Landauer's equation. ii) a resistance expression for nano conductors that is both voltage and temperature dependent is given for the first time in the literature and iii) the low voltage nonlinearity of the $I-V$ characteristics of ballistic nano conductors caused from the curvature of the Fermi-Dirac distribution function is discussed. It is concluded that there is an inescapable nonlinearity in the $I-V$ characteristics of nano conductors independent of the type and geometry of the conductor and this low voltage nonlinearity originates from the nature of the nano device namely the curvature of FermiDirac distribution functions at nonzero temperatures. Moreover, waveforms and THD of a sinusoidal signal passing through a ballistic nano conductor are obtained in HSPICE in order to demonstrate the effects of low voltage nonlinearity of ballistic nano devices. It is observed that THD decreases when the voltage amplitude increases in ballistic nano conductors as it is implied by the derived resistance expression.

\section{References}

[1] G. F. Close and H. S. P. Wong, "Assembly and electrical characterization of multiwall carbon nanotube interconnects", IEEE Trans. Nanotechnol. 7(5), 596-600 (2008). http://dx.doi.org/10.1109/TNANO. 2008.927373

[2] S. Kanthamani, N. Srujana Vahini and V. Abhaikumar, "Quasi-static modelling of carbon nanotube interconnects for gigahertz applications", Micro \& Nano Lett. 5(5), 328-332 (2010). http://dx.doi.org/10. $1049 / \mathrm{mnl} .2010 .0073$

[3] C. Xu, H. Li and K. Banerjee, "Modeling, analysis, and design of graphene nano-ribbon interconnects", IEEE Trans. Electron Devices 56(8), 1567-1578 (2009). http://dx.doi.org/10.1109/TED. 2009. 2024254

[4] H. Li, W. Y. Yin, K. Banerjee and J. F. Mao, "Circuit modeling and performance analysis of multi-walled carbon nanotube interconnects", IEEE Trans. Electron Devices 55(6), 1328-1337 (2008). http://dx.doi. org/10.1109/TED . 2008.922855

[5] S. Mohammadzadeh, D. Pouladsaz, R. Streiter and T. Gessner, "Electronic transport properties in copper nanowire", Microelectron. Eng. 85(10), 1992-1994 (2008). http://dx.doi.org/10.1016/j. mee.2008.06.005

[6] G. W. Hanson, "Fundamentals of Nanoelectronics", Prentice-Hall, New Jersey, USA, 2007.

[7] A. Naeemi, J. D. Meindl, "Compact physics-based circuit models for graphene nanoribbon interconnects", 
IEEE Transactions on Electron Devices 56(9), 18821833 (2009). http://dx.doi.org/10.1109/TED. 2009. 2026122

[8] S. Datta, "Quantum Transport: Atom to Transistor", Cambridge University Press, Cambridge, UK, 2005. http://dx.doi.org/10.1017/CB09781139164313

[9] C. Durkan, "Current at the Nanoscale: An Introduction to Nanoelectronics", Imperial College Press, London, UK, 2007.

[10] P. Avouris, "Carbon Nanotube Electronics", Proceedings of the IEEE 91(11), 1772-1884 (2003). http:// dx.doi.org/10.1109/JPROC. 2003.818338

[11] I. M. Kastnelson, "Minimal vonductivity in bilayer graphene", Eur. Phys. J. B 52(2), 151-153 (2006). http://dx.doi.org/10.1140/epjb/e2006-00294-6

[12] H. Ohnishi, Y. Kondo and K. Takayanagi, "Quantized conductance through individual rows of suspended gold atoms", Nature 395, 78-783 (1998). http://dx. doi.org/10.1038/27399

[13] P. J. Burke, "Lüttinger liquid theory as a model of the gigahertz electrical properties of carbon nanotubes", IEEE Trans. Nanotechnol. 1(3), 129-144 (2002). http://dx.doi.org/10.1109/TNANO.2002.806823

[14] Z. Xu, Q. S. Zheng and G. H. Chen, "Elementary building blocks of graphene-nanoribbon-based electronic devices", Appl. Phys. Lett. 90(22), 223115 (2007). http://dx.doi.org/10.1063/1.2745268

[15] Y. Mokrusov, G. Bihlmayer and S. Blugel, "Fullpotential linearized augmented plane-wave method for one-dimensional systems: Gold nanowire and iron monowires in a gold tube", Phys. Rev. B 72(4), 045402 (2005). http://dx.doi.org/10.1103/ PhysRevB.72.045402

[16] H. Kajiura, A. Nandyala, U. C. Coskun, A. Bezryadin, M. Shiraishi and A. Ata, "Electronic mean free path in as-produced and purified single-wall carbon nan- otubes", Appl. Phys. Lett. 86(12), 122106 (2005). http://dx.doi.org/10.1063/1.1885189

[17] S. Weingart, C. Bock, U. Kunze, F. Speck, Th Seyller and L. Ley, "Low-temperature ballistic transport in nanoscale epitaxial graphene cross junctions", Appl. Phys. Lett. 95(26), 262101 (2009). http://dx.doi. org/10.1063/1.3276560

[18] E. C. Walter, M. P. Zach, F. Favier, B. J. Murray, K. Inazu, J. C. Hemminger and R. M. Penner, "Metal nanowire arrays by electrodeposition", Chem. Phys. Chem. 14(2), 131-138 (2003). http://dx.doi.org/10. 1002/cphc. 200390022

[19] International Technology Roadmap for Semiconductors (ITRS) Roadmap, 2013, http://www.itrs.net/

[20] G. Rubio, N. Agrait and S. Vieira, "Atomic-sized metallic contacts: mechanical properties and electronic transport", Phys. Rev. Lett. 76(13), 2302-2305 (1996). http://dx.doi.org/10.1103/PhysRevLett.76.2302

[21] C. Z. Li, "Quantum transport in metallic nanowires fabricated by electrochemical deposition/dissolution", Appl. Phys. Lett. 72, 894-896 (1998). http://dx.doi. org/10.1063/1.120928

[22] J. A. Nesteroff, Y. V. Pershin and V. Privman, "Influence of nuclear spin polarization on quantum wire conductance", IEEE Trans. Nanotechnol. 4(1), 141-147 (2005). http://dx.doi.org/10.1109/TNANO. 2004.837855

[23] Y. Awano, S. Shintaro, M. Nihei, S. Tadashi, Y.Ohno and M. Takashi, "Carbon nanotubes for VLSI: interconnect and transistor applications", Proceedings of the IEEE 98, 2015-2031 (2010). http://dx.doi.org/ 10. 1109/JPROC. 2010.2068030

[24] A. Svizhenko, M. P. Anantram and T. R. Govindan, "Ballistic transport and electrostatics in metallic carbon nanotubes", IEEE Trans. Nanotechnol. 4(5), 557-562 (2005). http://dx.doi.org/10.1109/TNANO. 2005.851409 